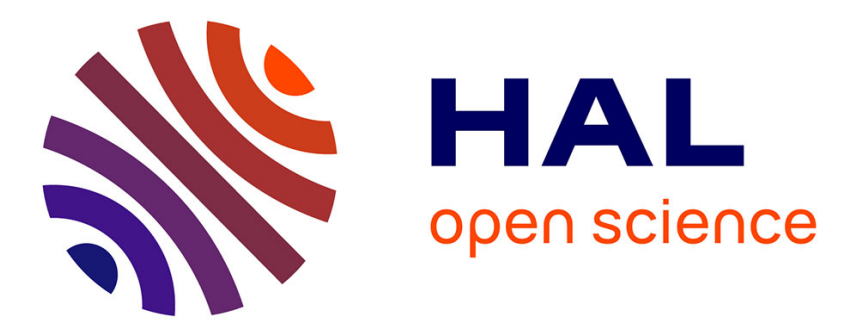

\title{
Giacomo Corneo, Le Capitalisme a-t-il fait son temps? Un voyage à travers les systèmes économiques alternatifs
} Audrey Dupont -Camara

\section{To cite this version:}

Audrey Dupont -Camara. Giacomo Corneo, Le Capitalisme a-t-il fait son temps? Un voyage à travers les systèmes économiques alternatifs. Lectures, 2019, 10.4000/lectures.37160 . halshs-03119474

\section{HAL Id: halshs-03119474 \\ https://shs.hal.science/halshs-03119474}

Submitted on 24 Jan 2021

HAL is a multi-disciplinary open access archive for the deposit and dissemination of scientific research documents, whether they are published or not. The documents may come from teaching and research institutions in France or abroad, or from public or private research centers.
L'archive ouverte pluridisciplinaire HAL, est destinée au dépôt et à la diffusion de documents scientifiques de niveau recherche, publiés ou non, émanant des établissements d'enseignement et de recherche français ou étrangers, des laboratoires publics ou privés. 


\title{
Giacomo Corneo, Le Capitalisme a-t-il fait son temps ? Un voyage à travers les systèmes économiques alternatifs.
}

\author{
Audrey Dupont - Camara \\ Doctorante en géographie / aménagement à l'université de Perpignan \\ (UMR ART-Dev)
}

$28 / 08 / 2019$

Le capitalisme actuel, combiné à l'économie de marché et à la propriété privée des moyens de production, est-il obsolète ${ }^{1}$ ? Pour Giacomo Corneo, spécialiste des finances publiques et des politiques sociales à l'université libre de Berlin, la seule critique des effets négatifs du capitalisme n'est pas satisfaisante, encore faut-il s'interroger sur quel serait le « meilleur » système à lui substituer. A travers un panorama anticonformiste des modèles économiques, où chaque chapitre explore une théorie économique « alternative ${ }^{2}$ spécifique, l'économiste passe en revue certaines propositions sociétales et analyse leur viabilité selon deux critères principaux : la coopération entre les personnes et l'allocation des ressources ${ }^{3}$. Il termine sa démonstration sur l'exposé du système qui serait selon lui le plus souhaitable : le socialisme actionnarial.

Pour se faire, Giacomo Corneo met en scène dans le prologue un père répondant aux critiques de sa fille qui dénonce les conséquences du système capitaliste : à savoir le gaspillage des ressources, l'injustice sociale et l'aliénation. Il l'invite alors à s'intéresser aux formes

${ }^{1}$ Le premier manuscrit est paru en allemand en 2014 aux éditions Goldegg, puis a été traduit en anglais sous le titre suivant: Is Capitalism Obsolete? A Journey through Alternative Economic Systems et publié en 2017 par Harvard University Press. Le texte en français a été remanié par Giacomo Corneo en intégrant un deuxième prologue (il s'agissait d'un appendice dans la version anglaise), ainsi que des références et notes bibliographiques complémentaires.

\footnotetext{
${ }^{2}$ L'un des enjeux principaux de l'ouvrage est finalement de proposer une définition stabilisée à ce qu'est une « alternative » économique.

${ }^{3}$ Selon l'auteur, la coopération entre les personnes constitue le cœur même de tout système économique ou politique que le système doit encourager être pérenne. L'allocation des ressources est définie par l'auteur comme «l'analyse précise décrivant qui produit quoi et comment et qui consomme quoi » (p.23).
} 
politiques et économiques qui se sont construites soit en complément du capitalisme pour en pallier les défauts, soit en rupture. Dans un second moment, l'économiste analyse les défaillances de l'État, que Platon avait proposé de résoudre à travers une société gouvernée par les rois-philosophes. Ce modèle implique une séparation radicale de la sphère économique et du politique, qui se ferait au détriment d'un fonctionnement démocratique ; une proposition récusée par l'auteur.

Dans le chapitre 3, Giacomo Corneo relit l'Utopie de Thomas Moore au regard du contexte sociétal actuel pour juger des applications concrètes d'une communauté de biens au XXIe siècle. Il en déduit que seules les normes sociales, l'altruisme et certaines valeurs intériorisées peuvent inciter les individus à coopérer entre eux. Mais sont-ils efficaces à l'échelle d'une société entière pour maintenir une telle communauté ? Le chapitre 4 s'attarde sur cette question en observant le rôle du commerce dans les relations interhumaines pour exposer de manière subjective les avantages (égalité matérielle, sécurité pour tous, relations plus chaleureuses) et inconvénients (ancrage local et diminution de l'horizon de vie des personnes, pression sociale et conformisme, contrôle étatique) de cette organisation sociale. Le chapitre 5 interroge l'attachement des citoyens à la consommation, à sa qualité et à sa diversité actuelle, à travers l'opposition entre "luxe et anarchisme ». Les théories de l'anarchiste Piotr Kropotkine sont exposées mais rapidement relayées au rang de sciencefiction. L'auteur estime en effet peu « sérieux » l'usage rationnel des ressources de la société en fonction des possibilités techniques, et moins des besoins humains.

Les pages suivantes nous semblent plus intéressantes car Giacomo Corneo s'attache à questionner les systèmes économiques contemporains, à commencer par le socialisme planifié, au chapitre 6. L'auteur teste la viabilité du système qui, par la complexité des procédures, l'opacité de l'information et le manque d'incitation économique, exige des personnes un sens du devoir hors du commun et irréaliste. Il avance ensuite l'idée que le marché représente la seule institution connue à ce jour capable de résoudre le problème de l'allocation des ressources dans un système économique complexe.

À partir du chapitre suivant, l'économiste analyse les modèles qui combinent le marché avec des éléments non capitalistes. Par exemple, l'autogestion est un modèle conçu pour pallier les défauts du capitalisme et de la planification sociale mais elle n'assure pas une 
répartition équitable des revenus des actifs et comporte des risques élevés de népotisme. Le socialisme de marché, présenté dans le chapitre 8, suppose que l’État soit propriétaire des moyens de production et que les entreprises soient autonomes par rapport à l'évolution du marché. Les managers seraient directement employés par l'État et n'auraient, selon l'auteur, que peu d'incitation pour maximiser les bénéfices.

De nouveaux modèles ont vu le jour pour répondre à ce problème de motivation : le socialisme de marché actionnarial (chapitre 9), le revenu universel et l'héritage social (chapitre 10) et l'économie de marché associé à l'État providence (chapitre 11). Le premier essaie de concilier mécanisme d'incitation à l'investissement par les entreprises privées et intervention du secteur social au moment opportun. Les seconds modèles se démarquent du précédent en ce qu'ils ne remettent pas en cause la propriété privée des moyens de production mais promettent une distribution «équitable » des ressources entre tous les membres de la société par un versement mensuel (revenu universel) ou par la réception d'un capital de départ à la majorité (héritage social). Enfin, la proposition d'un modèle tentant de joindre le capitalisme et l'État providence semble difficilement envisageable car, selon l'auteur, l'un et l'autre se rejettent mutuellement. Giacomo Corneo explique également que la loyauté envers la société et le principe de redistribution de la part des hommes politiques ne peuvent être garantis dans un tel système.

Ce tour d'horizon des alternatives économiques s'achève sur deux épilogues qui, reprenant la discussion entre père et fille, font le bilan des précédents chapitres et proposent un modèle alternatif cherchant à maximiser la cohésion sociale et la redistribution des richesses. Pour l'économiste et père de famille, le rôle de l'accroissement du capital public dans le système économique actuel est bel et bien de déjouer les fortes inégalités du capitalisme, qui menacent la prospérité partagée et la démocratie. Est-ce que la vocation de toute « alternative » au capitalisme, notamment celle du socialisme actionnarial - qu'il préfère, n'est-elle pas de rompre avec le cercle vicieux de la concentration des richesses et de la prise d'influence politique des capitaux privés pour garantir une meilleure égalité des chances ?

Si l'ouvrage est écrit dans un langage clair et organisé, il ne faut pas s'y tromper : il reste relativement difficile d'accès pour un novice de ces thématiques qui y chercherait une seule introduction aux différents modèles socio-économiques en Europe. Au contraire, 
Giacomo Corneo développe sa propre lecture argumentée de l'alternative économique et s'adonne à un exercice original dans le milieu académique. Son texte se rapproche d'un essai qui débat des limites et des réalités du capitalisme et envisage un modèle plus « social » de croissance économique. 